\title{
Von einer planlosen Überversorgung zur sektorübergreifenden Versorgungsplanung?
}

KARL-HEINZ SCHÖNBACH, HANS-GEORG FAUST

Karl-Heinz Schönbach ist Geschäftsführer im Geschäftsbereich Versorgung beim AOK-Bundesverband und Herausgeber der Zeitschrift „Gesundheits- und Sozialpolitik“"

Dr. Hans-Georg Faust ist politischer Koordinator im Geschäftsbereich Politik/ Unternehmensentwicklung beim AOK-Bundesverband und war von 1998 bis 2009 für die CDU Mitglied des Deutschen Bundestags

Allein aufgrund der demographischen Entwicklung wird eine bedarfsgerechte medizinische Versorgung in Deutschland nur noch sektorübergreifend und nach einem neuen Verständnis teamorientierter und interdisziplinärer Arbeitsteilung sicherzustellen sein. Für Doppelstrukturen fehlt allein schon das Personal. Und Sickerverluste an Sektorgrenzen sowie Kooperations- und Substitutionsverbote werden künftig unbezahlbar. Von daher müssen die ambulante „Bedarfsplanung" und die stationäre „Krankenhausplanung“ auf neue Grundlagen gestellt und verbunden werden. Die Fragen sind mit dem Versorgungsstrukturgesetz (VStG) aufgegriffen worden, aber weithin unbeantwortet geblieben. Das deutsche Gesundheitswesen hat es eben nicht leicht mit sich.

\section{Ambulante Bedarfsplanung}

Die Ausgangsfrage des VStG, wie die ambulante ärztliche Versorgung in strukturschwachen Gebieten künftig sichergestellt werden soll, ist weithin unbeantwortet geblieben. Aus vielzitierten Gründen ist der Beitrag einer „verteilenden Bedarfsplanung“ begrenzt, wenn die Überversorgung in den Städten weithin unberührt bleibt. Es braucht eine sektorübergreifende Sicherstellung, die die Krankenhäuser als deren Dienstaufgabe in die ambulante Sicherstellung einbezieht, wenn die Notwendigkeit dazu für den jeweiligen Bezirk auf geeignete
Weise festgestellt ist. Ebenso müssen Dritte, z.B. Kommunen, das Recht haben, Medizinische Versorgungszentren (MVZ) zu bilden, wenn eine offene Unterversorgung besteht. Die Länder etwa hatten an dieser Stelle zunächst klar Position bezogen. Und hatte nicht die Kassenärztliche Bundesvereinigung (KBV) zum Auftakt der Diskussion in seltener Offenheit vom Gebot einer „sektorübergreifenden Sicherstellung " gesprochen ${ }^{1}$ ? In der Diktion des VStG bleibt davon nur deklaratorisch ein Gremiengebilde nach $\mathbb{9} 90$ a SGB V ohne Aufgabenzuweisung bzw. Verantwortung: 
- Das gemeinsame Landesgremium kann zu sektorübergreifenden Versorgungsfragen Empfehlungen abgeben, wenn das Land es überhaupt konstituiert.

- Kommunen, die bei Unterversorgung MVZs gründen wollen, brauchen dafür die Zustimmung der nicht sicherstellungsfähigen Kassenärztlichen Vereinigung.

- Krankenhäuser können mitnichten per Dienstaufgabe planmäßig in die ambulante Sicherstellung einbezogen werden. Ihre Kapazitäten werden lediglich „gezählt“.

Die Bedarfsplanung bleibt mithin „ambulant“ statt „sektorübergreifend“. Sie kann aber nun nach Versorgungsstufen differenziert und auf adäquate Raumkategorien bezogen werden. Damit wird die Arbeit des Gemeinsamen Bundesausschusses an dieser Stelle nicht weiter rechtlich behindert. Das ist ein Fortschritt: Die Versorgungsstufen folgen dem Spezialisierungsgrad der Ärzte und den damit verbundenen Anforderungen an die Erreichbarkeit durch die Versicherten. Entsprechend geeigneten Raumkategorien werden Bedarfsmaße zugeordnet, während innerhalb der Räume Verteilungsmaße gesucht werden. So sollen für die hausärztlichen Funktionen „Mittelbereiche“ vorgesehen werden, die auch in anderen zentralen Sicherstellungsfragen der Daseinsfürsorge relevant sind, während die Verteilung der Sitze etwa auf die Gemeindeverbände erfolgt.

Die „Bedarfsplanung“ findet bisher auch keinen Weg zur „Versorgungsplanung“. Schon bisher wird ja dem Begriff zum Trotz bei der „Bedarfsplanung“ nicht der Bedarf geplant, sondern jedes weitere Angebot verteilt. Soll der Charakter einer „Versorgungsplanung“ ausgebildet werden, ist nicht länger allein nach der prinzipiellen Erreichbarkeit eines Arztes zu fragen, sondern auch nach dem „Versorgungsbedarf“ oder „Behandlungsbedarf“, der erfüllt werden soll. Während es sich die Einen an dieser Stelle tautologisch recht einfach machen, beginnt für die Anderen hier erst die Diskussion.

- Definitorisch einfach hat es der „Sprachgebrauch“ der Kassenärztlichen Vereinigungen, der die Summe ärztlicher Abrechnungspunkte je Versicherten als „Behandlungsbedarf" bezeichnet und der Einfachheit halber mit dem „Versorgungsbedarf“ gleichsetzt. Der Versorgungsbedarf der Versicherten - ob nach ärztlichen Leistungen oder Verordnungen - differiert dann zwar in medizinisch beunruhigender Weise stark nach den regionalen Abrechnungsgewohnheiten. Je weiter aber etwa die Abrechnungen pauschaliert werden, desto gleichförmiger kann der „Bedarf" ausgewiesen werden. Außerdem zählt ja nicht primär das, was der Versicherte in Anspruch nimmt oder die Krankenkasse diagnosengenau bezahlt, sondern das, was die Kassenärztliche Vereinigung nach ihrem ureigenen Verteilungsmaßstab nicht zuletzt zur Glättung der Einnahmenprofile an die Ärzte verteilt. Am Ende streiten sich bei diesem „Sprachgebrauch“ nur noch die verschiedenen Ligen der KVen um den „Versorgungsbedarf“ und seine unzureichende Konvergenz ${ }^{2}$, als müssten sie behandelt werden und nicht die Versicherten.

- Geht man weniger selbstreferenziell ans Werk, stehen die Leistungen im Mittelpunkt, die Versicherte brauchen. Bei den haus- und kinderärztlichen Leistungen, bei denen das Erkrankungs- und Behandlungspanorama abschätzbar ist und $\mathrm{Be}$ treuungsfunktionen im Mittelpunkt stehen, ergibt sich der Versorgungsbedarf weithin unter dem Gesichtspunkt der sicheren Verfügbarkeit und wohnortnahen Erreichbarkeit. Die Leistungen von Haus- und Kinderärzten sind deutlich weniger variabel und bedarfsgeprägter als fachärztliche Leistungen. Allein die Tatsche, dass erstere keine übermäßige Ausdehnung der PKV-Abrechnung und Igel-Leistungen kennen, verweist darauf, dass der Hausarzt macht, was er muss.

- Fachärzte würden für sich auch gar nicht zurückweisen, dass sie machen, was sie können. Ihr PKV-Anteil ist in einigen Fachgruppen ökonomisch stark hypertroph ${ }^{3}$. PKV-Versicherte weisen der Sprachregelung der KVen nach offenbar einen mehrfach erhöhten fachärztlichen Versorgungsbedarf auf. Wenn es aber tatsächlich abzuschätzen gilt, was der Bedarfshintergrund der Leistungen ist, wird nach den zugrunde liegenden Diagnosen zu fragen sein. Ein solcher Diagnosebezug ist für die GKV allerdings in hohem Maße gestört. Zum einen fehlt es weithin an der gezielten $\mathrm{Zu}$ weisung des Hausarztes (Zielauftrag) - ein Primärarztsystem wird geradezu abgelehnt, zum anderen wird eine diagnosebezogene Abrechnungsdokumentation mit Hilfe von vorbereiteten Kodierrichtlinien bis heute unterbunden ${ }^{4}$. Somit kann die Abbildungsleistung des ambulanten Bewertungsmaßstabes ärztlicher Leistungen für die Fachärzte kaum ein zutreffendes Bild für den Behandlungsbedarf bzw. Versorgungsbedarf geben.

Die sich bei dieser Ausgangslage ergebenden Fronten laufen quer durch die Lager. So hatte die Kassenärztliche Bundesvereinigung parallel $\mathrm{zu}$ ihrem o. g Programmpapier ein Reformpapier vorbereitet, das zumindest mit Wahltarifsoptionen der Versicherten den Übergang zu einem Primärarztsystem $^{5}$ vorsah. Für die Einen lag hierin die Zukunftssicherung der ambulanten fachärztlichen Versorgung, die sich nun weitgehend dem Kern ihres Versorgungsauftrages widmen können sollte. Die Anderen betrachten jede Form der Strukturierung des primären Patientenzugangs als indiskutabel, um sich dann umso hingebungsvoller der Grenzen zur ambulanten Behandlung im Krankenhaus zu widmen. Für beide Lager scheint

1 KBV: Die Zukunft der vertragsärztlichen Versorgung, Vorschläge zur Weiterentwicklung der ambulanten Medizin, Manuskript 25. November 2009.

2 FALK lehnt weitere bundesweite HonorarUmverteilung ab: Politische Probleme nicht auf Kosten der Patienten und Ärzte lösen: Gemeinsame Pressemeldung der KVen aus Bayern, Baden-Württemberg, Hessen und Mecklenburg-Vorpommern vom 16. Mai 2011

3 Nach Daten des Statistischen Bundesamtes erzielen Haus- und Kinderärzte rund 85 Prozent ihrer Praxiseinnahmen aus Umsätzen für Versicherte der Gesetzlichen Krankenversicherung, was deren Versichertenanteil nahe kommt, während der Anteil bei Facharztgruppen bis unter 50 Prozent sinken kann. Vgl. Stat. BA: Kostenstruktur bei Arztpraxen 2007, Fachserie 2, Reihe 1.6.1.

4 Die schwarz-gelbe Koalition hat die Kodierrichtlinien auf Druck der KVen mit dem VStG 2012 gesetzlich suspendiert, obgleich die KBV sich ursprünglich gegen eine solche Suspendierung gewandt hatte.

5 Vgl. KBV: Überlegungen zu einer wettbewerbsorientierten Neustrukturierung der Versorgungsebenen, Manuskript, 15. Mai 2008. 
der Kern des Versorgungsauftrages aber in hohem Maße von der jeweils für den Patienten gelten Gebührenordnung bestimmt zu sein. Aus dieser Sicht wäre die vom Verband der privaten Krankenversicherung beklagte Überbehandlung der privat Versicherten ebenso „bedarfsgerecht" und medizinisch geboten wie die fachärztliche Zurückhaltung und Terminflucht bei gesetzlich Versicherten. Der Präsident der Bundesärztekammer hat sich inzwischen auf das Argument eingelassen, dass auch Ärzte halt ökonomischen Anreizen folgen. Die Regeln der Profession schützen ein ethisches Verhalten der Ärzte offenbar nicht mehr hinreichend gegenüber ökonomischen Anreizen. Von daher verantwortet ein Staat, der zwei unterschiedliche Gebührenordnungen zur Geltung bringt, politisch ein Auseinanderfallen der medizinischen Ethik. ${ }^{6}$ Daher steht inzwischen eine privatärztlich wie vertragsärztlich „einheitliche Gebührenordnung“ auf der Tagesordnung ${ }^{7}$ : Kaum eine Facharztgruppe, die angesichts dieser Debatte nicht in Harnisch geriete.

Übergreifend populär werden dagegen ärztliche Betrachtungen zum Versorgungsbedarf aufgenommen, die aus soziographischen Merkmalen mit multiplem Regressionsaufwand höhere Versorgungsbedarfe ableiten, um den Arztmangel so vom Lande auch in die Verdichtungsgebiete zu exponieren ${ }^{8}$. Die Zahl der von Arbeitslosigkeit Betroffenen, der Ehelosen und Ein-PersonenHaushalte, der Pflegebedürftigen, der Hochbetagten usw. lassen sich statistisch heranziehen, um den Versorgungsbedarf zu beflügeln. Punktmengen aus Abrechnungsstatistiken sind jeder mathematischen Behandlung zugänglich. Die Regionalisierung des Versorgungsbedarfes trifft sich dann gelegentlich mit Argumenten zur Regionalisierung des morbiditätsorientierten Risikostrukturausgleichs, kurzum: eine interessen-, keine ordnungsgeleitete Diskussion.

\section{Anknüpfung an den Diagnosebezug}

Die kardinale Voraussetzung, um ärztlichen Abrechnungen eine Relevanz für die Bemessung des Behandlungs- bzw. Versorgungsbedarfs der Versicherten abgewinnen zu können, scheint daher ein Anknüpfen eben dieser Abrechnungen an die medizinischen Bedarfe der Versicherten selbst zu sein. Fehlt der Diagnosebezug, fehlt die Bedarfsorientierung. Es bedarf mithin einer patienten- bzw. diagnosebezogenen Gebührenordnung ambulanter (fach-) ärztlicher Leistungen. Erst wenn auch die Abrechnung den ärztlich definierten „Behandlungsbedarf“" vom Versicherten her definiert, wird er logisch stringenter

\section{Punktmengen aus Abrechnungsstatistiken sind jeder mathematischen Behandlung zugänglich.}

mit einem nicht nur ärztlich definierten „Versorgungsbedarf“ der Versicherten verbunden werden können. Wird die Gebührenordnung im notwendigen Fall mit Prozeduren hinterlegt und nach Schweregraden differenziert, hilft sie zudem, dass die (fach-) ärztliche Leistung den Versicherten nach medizinischen (Bedarfs-) Kriterien erreicht. Eine verrichtungsbezogene Gebührenordnung, die der Arzt im Nachhinein anhand von Kodierrichtlinien begründet, reicht für eine logische Verbindung nicht aus.

\section{Verknüpfungsaspekte ambulant - stationär}

Naturgemäß wird eine medizinisch begründete, diagnosebezogene Gebührenordnung auch durchgehend betriebswirtschaftlich kalkuliert werden müssen. Es verbietet sich für eine wissenschaftlich fundierte Disziplin mit organisierter Professionalität, Gebührenrelationen „verrichtungsbezogen“ zu definieren und ihr wertmäßiges Verhältnis auf unzureichender kalkulatorischer Basis zu verhandeln. Ebenso verbietet es sich, die Gebührenordnung nach dem „Ort“ der Leistungserbringung oder der Rechtsform, deren sich ein Leistungserbringer bedient, zu unterscheiden. Die (berechtigte) Empörung, dass ein und dieselbe Leistung im Krankenhaus teurer oder weniger teuer ist als in ambulanter Praxis, verkommt als jahrzehntelange Dauererregung zur berufspolitischen Posse. Kurzum: Die neue diagnosebezogene Gebührenordnung muss die ambulante Behandlung im Krankenhaus einschließen.

Der Ansatz des VStG, neben das ungebremst, ökonomisch induziert wach- sende ambulante Operieren einen von Planung und Verträgen vollends losgelösten Bereich der spezialfachärztlichen Versorgung zu stellen, ist allein aufgrund des schwergängigen Richtlinienvorlaufs soweit zurück geblieben, dass er wie frühere Modellierungen des $\mathbb{1} 116 \mathrm{~b}$ SGB V Makulatur bleiben dürfte9. Soweit wie die ambulante Behandlung inzwischen im Krankenhaus vorgedrungen ist und die Versorgungswirklichkeit bestimmt, braucht es eine ordnungspolitische Harmonisierung: Rund die Hälfte der Patienten, die heute mit dem Krankenhaus in Berührung kommen, erhält keine Behandlung „am Bett“. Formen und Anlässe der ambulanten Behandlung im Krankenhaus wurden über Jahrzehnte ad hoc geschaffen, ohne dass zumindest im Nachhinein ein stimmiges Konzept entstanden wäre. Von der Bedarfsplanungs-, Vertrags-, Vergütungs-, Mengensteuerungs- und Qualitätssicherungsseite her ist der entstandene Flickenteppich mit der nächsten Reform konzeptionell als „sektorunabhängige Versorgung “ zu ordnen. Heute bestehen nebeneinander ${ }^{10}$ :

- Vor- und nachstationäre Behandlung im Krankenhaus ( $\$$ 115a SGB V ),

- teilstationäre Behandlung im Krankenhaus ( $\int 39$ SGB V),

6 Die SPD hat daraus auf ihrem Leipziger Parteitag die Forderung nach einer „einheitlichen Gebührenordnung, abgeleitet. Vgl. Beschluss des Parteitages vom 26. September 2011, Solidarische Gesundheitspolitik für alle Bürgerinnen und Bürger.

7 Spahn für Reform der privaten Krankenversicherung („Diese Trennung ist nicht mehr zeitgemäß, Sie finden dafür nicht einmal mehr auf einer CDU-Mitgliederversammlung eine Mehrheit.“), in: ärzteblatt.de, Mittwoch, den 12. April 2012.

8 Vgl. Stillfried, Dominik Graf von, Czihal, Thomas: Möglichkeiten der fachgruppenspezifischen Risikoadjustierung der Verhältniszahlen für eine zeitgemäße Versorgungsplanung, in Gesundheits- und Sozialpolitik, Heft 2 in 2011, S. 29 ff.

9 So ließ sich der Vorsitzende der KBV bei einem Fachsymposium in München vernehmen, vor Mitte 2014 könne nicht mit der operativen Umsetzung der neuen gesetzlichen Regelung des Paragrafen 116b SGB V gerechnet werden,. Es sei noch völlig offen, wie der neue Versorgungsbereich ausgestaltet und wie die Grenze zur ambulanten Regelversorgung gezogen werden sollen. Vgl. Ärztezeitung vom 26. Juli 2012.

10 Vgl. Leber, Wulf: § 115x - Spezialärztliche Versorgung: Ein Reformprojekt ab 2011, in F\&W, Ausgabe 6 in 2010, S. 10 ff. 
- die Hochschulambulanzen (\$S 117 SGB V),

- das ambulante Operieren $(\mathbb{S} 115 \mathrm{~b}$ SGB V),

- die Behandlung von seltenen Erkrankungen und Krankheiten mit besonderem Krankheitsverlauf (\$ 116b SGB V),

- die psychiatrischen Institutsambulan-

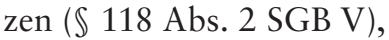

- die Sozialpädiatrischen Zentren (\$ 119 SGB V),

- die pädiatrischen Spezialambulanzen ( $(120$ Abs. 1a SGB V),

- ambulante Ermächtigungen von Krankenhausärzten (\$116 ff. SGB V)

- und ambulante Versorgung bei Unterversorgung ( $\mathbb{1 1 6}$ a SGB V ). ${ }^{11,12}$

Die Formen der ambulanten Behandlung im Krankenhaus sind nicht nur schrittweise zu überprüfen, sondern bei Fortbestand auch in einer durchgehenden Systematik einschließlich der entsprechenden vertragsärztlichen Leistungen wettbewerbsgleich zu einer sektorunabhängigen Versorgung zu verbinden ${ }^{13}$. Die Einordnung kennzeichnet Abb.1:

1. Danach verblieben die hausärztliche und allgemeine fachärztliche Versorgung in der Obhut der ambulanten Bedarfsplanung und der Finanzierung nach Prinzipien der vertragsärztlichen Vergütung. Geplant werden kann hier wohnortnah nach Arztsitzen

- Die hausärztliche Vergütung erfolgt weiter innerhalb der separaten Gesamtvergütung tendenziell nach Fallpauschalen und Zuschlägen insbesondere für chronische Erkrankungen.

- Bei den Abstaffelungsgrenzen für Einzelleistungen sollte die durchschnittliche Morbidität in der Praxis berücksichtigt werden.

2. Die stationären Leistungen, die notwendigerweise unter Zuhilfenahme des Bettes zu erbringen sind, verblieben in der Krankenhausplanung der Länder und unter Finanzierung der DRGs.

- Der notwendige Übergang zu einer monistischen Krankenhausfinanzierung bedingt den entsprechenden Übergang zu einer stationären „Rahmenplanung“.

- Wir werden an späterer Stelle in

Abbildung 1: Einordnung einer sektorunabhängigen Versorgung

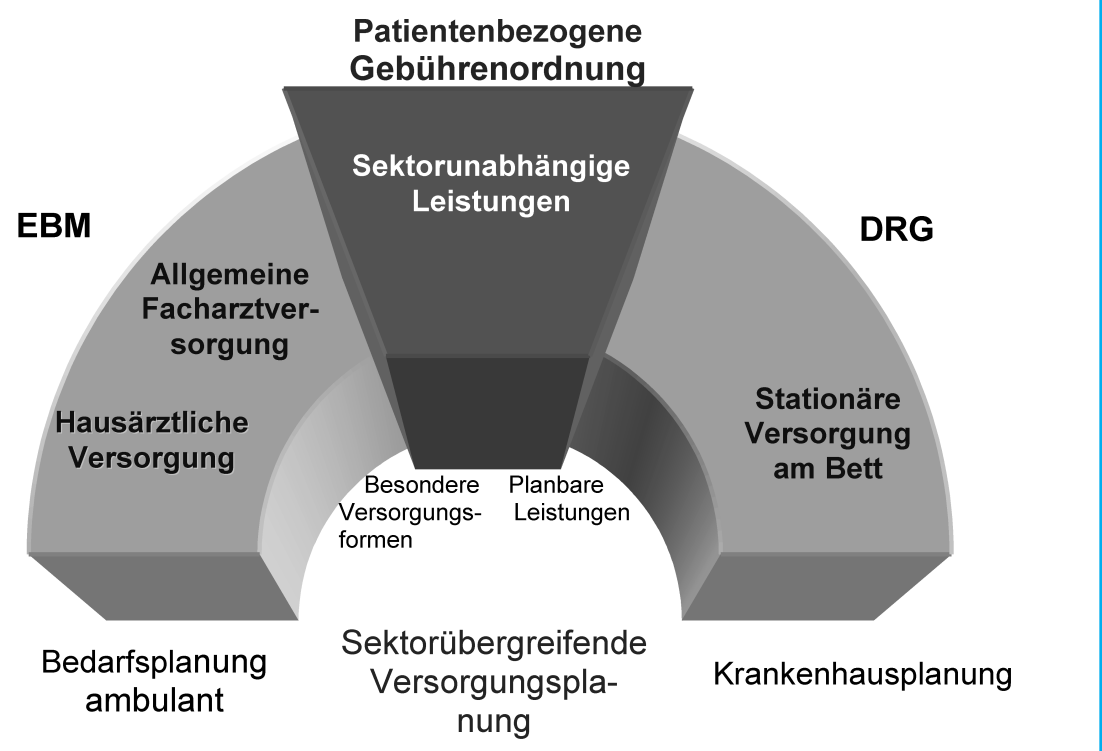

Eigene Darstellung

diesem Beitrag ausführen, dass eine Rahmenplanung zumindest für stationäre Routineleistungen und planbare Eingriffe angezeigt ist. $^{14}$

3. Und die Leistungen der „sektorunabhängigen Versorgung“ werden nach einer neuen, schrittweise aufzubauenden „patientenbezogenen“ Gebührenordnung vergütet. In der dafür vorzusehenden sektorübergreifenden Versorgungsplanung wird sektorunabhängig die Verfügbarkeit von Leistungen geplant.

Für „sektorunabhängige Leistungen“ wird eine durchgehend kalkulierte, am Patientenproblem orientierte, d.h. diagnosebezogene Gebührenordnung, die im notwendigen Fall nach Schweregraden differenziert, institutionelle, vertragsrechtliche und honorartechnische Sonderregelungen für zahlreiche Sonderformen der ambulanten Erbringung im Krankenhaus entbehrlich machen. Sie werden ebenso die entsprechenden fachärztlichen Leistungen der Vertragsärzte chancen- und wettbewerbsgleich einbeziehen. Voraussetzung ist, dass die Gebührenordnung sektorübergreifend legitimiert wird - daraus folgt als Szenario für die Sektorunabhängige Zukunft:

1. Auftraggeber der sektorübergreifenden ambulanten Gebührenordnung ist dementsprechend der Gemeinsame
Bundesausschuss (G-BA), in dem alle Sektoren repräsentiert sind.

2. Die sektorübergreifende Gebührenordnung wird dann auch vorzugsweise durch die Arge aus InEK und InBA erstellt. Kommt eine Entscheidung dazu im G-BA nicht zustande, hat der BMG ein Ersatzvornahmerecht.

3. Diese Gebührenordnung gilt für die Krankenhäuser auf gesetzlicher Grundlage unmittelbar. Für die Vertragsärzte gewinnt sie durch Einbezug in den Bundesmantelvertrag Geltung.

11 Nur der Vollständigkeit halber: Weitere Formen der ambulanten Behandlung sind Disease-Management- Programme ( $\$ 137 \mathrm{f}$ SGB V), Belegärzte ( $\$ 121$ SGB V) und Medizinische Versorgungszentren (§ 95 SGB V).

12 Zukünftig wird diese Vielfalt ambulanter Klinikleistungen noch mit dem am 01.01.2013 in Kraft tretenden Gesetz zur Einführung eines pauschalierenden Entgeltsystems für psychiatrische und psychosomatische Einrichtungen (PsychEntgG) ergänzt durch: (a) die psychiatrischen Institutsambulanzen an psychosomatischen Einrichtungen (§ 118 Abs. 3 SGB V)und (b) die geriatrischen Institutsambulanzen (§ 118a SGB V).

13 Vgl. Schönbach, K. H.: Sektorübergreifende Sicherstellung mit Vertragspflicht der Krankenkassen, in: Gesundheits- und Sozialpolitik, S. 11 - 18 sowie in: Jacobs, K., Schulze, S. Sicherstellung der medizinischen Versorgung, Berlin (kompart)2011, S. 97 ff.

$14 \mathrm{Vgl}$. Abschnitt „Krankenhausplanung“. 
Auf diese Weise könnten über die vertragsärztliche Versorgung hinaus fachärztlichen Kapazitäten der Krankenhäuser systematisch in die ambulante Behandlung einbezogen werden. Ob, wann und inwieweit sie in die ambulante Behandlung einbezogen werden, könnte

\section{Sektorübergreifende Bedarfs- planung wird sich künftig auf einen diagnosebezogenen Leistungsbegriff beziehen müssen.}

gen sektorunabhängig zu kontrahieren. Zweifellos hat der Staat bei besonderen Gütern, wie sie die Leistungen der sozialen Krankenversicherung bilden, eine Fülle von Normen für Qualität, Sicherheit und Transparenz vorzugeben. Innerhalb der gesetzlichen und untergesetzlichen Normen stehen sich bei der Realisierung der Versorgung allerdings zwei „Welten“ gegenüber ${ }^{15}$ :

„Alte Welt": Im tradierten deutschen Gesundheitssystem mit staatlich dominiertem Krankenhaussektor und von Verbänden

Gegenstand der ambulanten bzw. sektorübergreifenden Bedarfsplanung sein. Allein von daher verbieten sich Konstruktionen sektorübergreifender Gremien etwa nach $\mathbb{S} 90$ a SGB $\mathrm{V}$, bei denen die (auch) ökonomisch verantwortlichen Partner nicht in Parität gegenüberstehen und das Land nicht ohne Beeinträchtigung seiner Aufsichtsfunktion nachkommen kann. Anhörungsrechte Dritter bleiben davon unberührt.

Eine sektorübergreifende Bedarfsplanung wird sich künftig auf einen gleichnamigen, diagnosebezogenen Leistungsbegriff beziehen müssen, wie er mit einer neuen Gebührenordnung entstehen soll. Insoweit ist die geforderte patientenbezogene Gebührenordnung ein konstitutiv notwendiges Instrument der Sicherstellung. Es bedarf dann auf entsprechend sektorunabhängiger empirischer Grundlage bzw. Grundlage einer Versorgungsberichterstattung angemessener Richtlinien des G-BA zu einer sektorunabhängigen Bedarfsplanung. Dabei sind naturgemäß in erster Linie ambulante Krankenhauskapazitäten dort systematisch einzubeziehen, wo die ambulante Versorgung durch Vertragsärzte unterhalb hinreichender Bedarfsgrade bleibt.

Ein in dieser Weise korporatistisch ausgestattetes Szenario ,sektorübergreifender Bedarfsplanung“ dürfte sich noch innerhalb der Grenzen des in der deutschen Gesundheitspolitik durchaus Realisierbaren befinden. Andererseits bedürfte es nur einer gesetzlichen „Vertragspflicht“ der Krankenkassen (also ihrer Sicherstellung), auf der Grundlage einer morbiditätsorientierten Versorgungsplanung unter der Rechtsaufsicht der Länder die erforderlichen Leistun- unter Einigungszwang mit der Hilfe von Schiedsämtern geschlossenen Verträgen

- erfolgt die „Planung“ zentral nach dem jeweiligen „Angebot“,

- erfolgt der „Marktzugang“ durch öffentlich rechtliche „Zulassung“,

- wird der „Bedarf“" nach den jeweils erbrachten „Leistungen“ definiert

- und entstehen „Verträge“ unter „Kontrahierungszwang“,

- d.h., die vertragliche Allokationsleistung ist maximal eingeschränkt.

„Neue Welt": In einem marktförmig koordinierten Gesundheitswesen, das „Sektoren“ überwunden hat oder zumindest schrittweise überwinden will

- erfolgt die Planung dezentral nach der „erwarteten Nachfrage“,

- ist der Marktzugang im Rahmen gesetzlicher Vorschriften frei,

- konkurrieren die Anbieter um die größte Nachfrage, die der Bedarf determiniert,

- und entstehen „Verträge“ unter Wettbewerbsrecht,

- d.h., die vertragliche Allokationsleistung der Verträge ist hoch.

Wird nun, weil die ambulante Sicherstellung sektorisoliert zu scheitern droht, eine sektorübergreifende Sicherstellung erforderlich, stellt dies zweifellos - wie nun auch der Sachverständigenrat unumwunden gutachtet - Lösungen aus der „neuen Welt“ eines „marktförmig koordinierten“ Gesundheitswesens auf die Tagesordnung: Eine Verbindung tradierter ambulanter und stationärer Ordnungsprinzipien zu einem „Dritten Sektor“, wäre purer Eklektizismus zu
Lasten der Patienten und weder medizinisch, noch ökonomisch zu vermitteln. Allein die Literaturliste zum Einweisungs- und Entlassmanagement für einen „Dritten Sektor“ mutete makaber an. Aufgerufen ist mithin der schrittweise Übergang zur Marktsteuerung unter den Rahmenbedingungen der sozialen Krankenversicherung ${ }^{16}$.

\section{Übergang zu einem Einzelvertragssystem}

Gerade in dem geschilderten Sinne hat der Sachverständigenrat zur Begutachtung der Entwicklung im Gesundheitswesen (SVR) zur Versorgung an der Schnittstelle zwischen ambulanter und stationärer Versorgung Stellung genommen ${ }^{17}$. Er verbindet mit dem Einstieg des VStG einer „spezialfachärztlichen“ Versorgung gerade die Konzepte, die die Koalition bei dieser Diskussion ignoriert hatte, nämlich die oben angeführte

- schrittweise Neuordnung der ambulanten Behandlung im Krankenhaus ${ }^{18}$ mit der

- sektorunabhängigen Einführung einer diagnosebezogenen Gebührenordnung ${ }^{19}$.

Das Fazit aus den Überlegungen zu einer sektorübergreifenden Sicherstellung mit Vertragspflicht der Krankenkassen lautet:

„Ein sektorübergreifender Wettbewerbsansatz, der die Sicherstellung der ambulanten Versorgung garantiert, die ambulanten Krankenhausleistungen einbezieht und kalkulierbare Vergütungen sichert, ist möglich. Statt des Kontrahierungszwangs mit jedem Anbieter muss es dabei

15 Vgl. Schönbach, K.H.: a.a.O., S. 15 (Vertragsprinzip statt Kontrahierungszwang).

16 Die soziale Krankenversicherung findet an dieser Schnittstelle zu „Managed Competition"( Vgl. Enthoven, A.C.: Theory and Practice of Managed Competition, 1988) mit den Merkmalen: Einheitlicher Leistungskatalog, Versicherungspflicht der Bürger, einkommensabhängige Prämien mit Risikostrukturausgleich (M-RSA), freier Marktzutritt für Leistungsanbieter, vollständige Geltung des Kartellrechts ohne Kontrahierungszwang der Krankenkassen, monistische Finanzierung auch der stationären Versorgung.

17 Vgl. SVR, Sondergutachten 2012, Kurzfassung, TZ 183 ff. zu den Wettbewerbsbedingungen an der Sektorengrenze zwischen ambulant und stationär.

18 Vgl. Leber, Wulf, Fußnote 8.

19 Vgl. Schönbach, K.H.: Fußnote 9. 
eine leistungsbezogen sicherstellende Vertragspflicht der Krankenkassen geben. Statt der Zulassung aller potentiellen Anbieter und ihrer kollektiven Regulierung im Nachhinein wird die Einführung des Vertragsprinzips zu mehr Qualität und Verlässlichkeit auf der Mikroebene der Versorgung führen und die Management-Unterstützung der Ärzte nachdrücklich verbessern können“. ${ }^{20}$

Diese sieht auch der Sachverständigenrat, wenn er die Umsetzung seines Konzepts an dieser Stelle einer Konkretisierung durch „selektivvertragliche Regelungen“ vorbehält (TZ 181), mit denen eine sektorunabhängige Gebührenordnung (aus Relativgewichten) im Wettbewerb verpreist wird. Damit schlägt er den fehlenden Schritt vor, „... der den Wettbewerb der Leistungserbringer um Patienten auch zu einem Wettbewerb der Krankenversicherer um gute Versorgung und damit letztlich auch um Versicherte werden lässt“ (TZ 183).

Ein Minderheitenvotum wird bei der Beschlussfassung im SVR an dieser Stelle nicht kenntlich. Das gilt im Übrigen auch für die Ausführungen zum Schutz des Wettbewerbs und zu einem „versöhnlichen Miteinander von Kartell- und Sozialrecht“ (TZ 39). Zum einen seien die Sozialbücher dem GWB gegenüber spezieller und genössen daher Vorrang. Zum anderen griffen kartellrechtliche Ge- und Verbote nur in solchen Bereichen, in denen den Krankenkassen Handlungsspielräume eröffnet seien: „Sind die Krankenkassen gesetzlich zu einem bestimmten Verhalten verpflichtet, so kann dies keine wettbewerbsrechtlichen Sanktionen nach dem GWB hervorrufen, weil der Freiraum fehlt, den wettbewerbliches Verhalten voraussetzt" ${ }^{21}$ Fraglich ist, ob der SVR das Infektionsrisiko einer Anwendung des GWB auf die Rechtsprechung des EuGH zur (nicht nur funktionalen) Unternehmenseigenschaft der Krankenkassen zutreffend einschätzt. Führte die subkutane Anwendung des nationalen Kartellrechts dazu, dass der EuGH die Krankenkassen nicht länger als Körperschaften zu rein sozialen Zwecken einordnet, könnte aus einer künftigen EuGH-Rechtsprechung diejenige Sprengkraft für die Soziale Krankenversicherung erwachsen, die Interessenten einer Privatisierung suchen. Die Anknüpfung der 8. GWB Novelle bei den leitenden Grundsätzen des SGB V verrät solche Ansinnen. Dies sollte der Rat bedenken.

Gleichwohl Chapeau! Ein bemerkenswertes Gutachten! Wird es um die Überlegungen ergänzt, die der SPDLeitantrag $^{22}$ zur Vereinheitlichung der Versicherungssysteme und einer „einheitlichen Gebührenordnung “ für GKV und PKV ausführt, scheint eine reformträchtige Legislaturperiode vorbereitet, zumal sich in der Partei der GRÜNEN und der CDU/CSU parallele Überlegungen finden, mal mit dem Akzent der Bürgerversicherung, mal in Richtung auf eine Neuordnung des Wettbewerbs zwischen GKV und PKV.

\section{Sektorunabhängige Versorgung aus Perspektive der Krankenhäuser}

Auch aus Sicht der Krankenhäuser besteht die Notwendigkeit einheitlicher Rahmenbedingungen für Vertragsärzte und Krankenhäuser. Dies zeigt allein das Beispiel des „ambulanten Operierens“. Noch hat der Gesetzgeber gezögert, diesen Bereich in die Regelungssystematik des neuen $\mathbb{1} 116 \mathrm{~b}$ SGB V aufzunehmen. Aus gutem Grund - hätte doch ohne ein stimmiges Konzept zur Koordinierung

Der Sachverständigenrat hat ein bemerkenswertes Gutachten vorgelegt: Chapeau!

eine sektorübergreifende Budgetierung von Leistungsmengen ins Haus gestanden, das ominöse „Dritte Budget“.

Die bestehende Regulierung krankt insbesondere an den Fehlanreizen der Vergütung. So ist es für die Krankenhäuser lukrativer, Kapazitäten im stationären Sektor mit Kurzzeitfällen auszulasten, als die Leistungen ambulant anzubieten. Vom Sachverständigenrat wird konkret vorgeschlagen, an das bestehende DRG-System anzuknüpfen und die Vergütung für die eigentliche vergleichbare Leistung durch Abzug von pauschalisierten Ist-Kosten für z. B. Übernachtung und Überwachung zu ermitteln. Damit wird auch die notwendige Abgrenzung zwischen vollsta- tionären Operationen, Ein-Tages-Fällen und ambulanten Operationen gelöst ${ }^{23}$. Auf der Grundlage einer solchen patientenbezogenen, aus der DRG-Systematik abgeleiteten Vergütung soll sich der Wettbewerb zwischen Krankenhäusern, MVZs, Vertragsärzten und ambulanten Operationszentren auf der Basis von Selektivverträgen entwickeln

\section{Krankenhausplanung}

Die Einbeziehung der Krankenhäuser bei einer sektorunabhängigen Versorgung ist im Sinne des Wettbewerbs zu begrüßen und im Fall der regionalen ambulanten Unterversorgung unverzichtbar. Allerdings wird damit für Regionen mit Überversorgung ein zusätzliches Angebot geschaffen, womit sich konsequenterweise die Frage stellt, ob das Krankenhaus nur im Bereich des ambulanten Angebots überflüssig ist oder generell mit Blick auf die Krankenhausplanung ein Überangebot besteht.

Nach unserer Verfassung ist die Sicherstellung einer angemessenen medizinischen Versorgung eine wichtige Aufgabe öffentlicher Daseinsvorsorge, die auf dem Sozialstaatsprinzip beruht. Daraus leiten die Länder ihre Verantwortung für die Krankenhausplanung und die Standorte ab: Es ist ein Gebot der Ehrlichkeit, dass man, bevor man sich den versorgungstechnischen Fragen der Krankenhausplanung nähert, den krankenhauspolitischen Bereich betrachtet, der mit Versorgung der Patienten wenig, mit regionalen gesellschafts- und wirtschaftspolitischen Kräften aber viel zu tun hat. So ist das örtliche Krankenhaus „Arbeitgeber“, „Einkäufer“" von Dienstleistungen und Waren, „werthaltige Immobilie“, „Aushängeschild“ für die Kommune und die Kommunalpolitiker und vieles andere mehr. Diese vielfältigen Aspekte werden „politisch“ zur Geltung gebracht, wenn strukturelle Änderungen der regionalen Krankenhauslandschaft anstehen oder gar ein Krankenhaus geschlossen werden soll.

Die bestehende Krankenhauslandschaft beruht mehr oder weniger (in

20 Schönbach, K.H. ebenda, als Fazit, vgl. S. 17. 21 SVR Sondergutachten, TZ 39.

22 Vgl. Fußnote 6.

23 Vgl. SVR, Sondergutachten 2012, Langfassung S. 290. 
den neuen Ländern) auf dem althergebrachten Standortangebot, das bei seiner historischen Etablierung von inzwischen weithin abgelegten medizinischen und versorgungstechnischen Grundsätzen geprägt war: So war die Medizin vor 50 Jahren weit weniger spezialisiert, weniger geräteaufwendig, eher pflege- als funktionsorientiert und das Krankenhaus spielte bei der Notfallerstversorgung eine wichtige, häufig entscheidende Rolle. Diese Aufgabe der Erstversorgung übernehmen heute die Notarztsysteme. Mithin ist die bestehende Krankenhausdichte in erster Linie historisch mit der Notwendigkeit der schnellen medizinischen Notfallversorgung erklärbar.

Die Bundesländer müssen nach $\mathbb{S} 6$ KHG zur Gewährleistung einer bedarfsgerechten Versorgung der Bevölkerung Krankenhauspläne aufstellen, wobei die Einzelheiten in Krankenhausgesetzen der jeweiligen Bundesländer geregelt werden. Zahl und Art der Krankenhausbetten orientieren sich an Kennzahlen wie der Bevölkerungszahl, der Verweildauer im Krankenhaus und

\section{Die Länder sollen sich auf die Rahmenplanung konzen- trieren, während die konkrete Zulassungs- und Vertrags- hoheit auf die Krankenkassen übergeht.}

der Bettenauslastung ${ }^{24}$. Damit wird der Bedarf der Vergangenheit normativ festgelegt und fortgeschrieben, ohne den tatsächlichen Versorgungsbedarf zu berücksichtigen. Jedenfalls war durch die bisherige Planung nicht zu verhindern, dass trotz deutlich gesunkener Krankenhaus- und Bettenzahlen sowie bei verkürzten Verweildauern die durchschnittliche Kapazitätsauslastung seit Einführung der DRGs bei kaum mehr als $78 \%$ liegt.

Zwar unternehmen auch einzelne Bundesländer Anstrengungen, die Krankenausplanung zu qualifizieren und Qualitätsindikatoren in den Krankenhausplan aufzunehmen ${ }^{25}$. Die allgemeine Entwicklung und der Bedarf, mit den Krankenhäusern über die Landeskrankenhausgesellschaften hinaus zu übergreifenden Vereinbarungen zu kommen, lassen allerdings eine stärkere bundesweite Regulierungsvorgabe des G-BA als geraten erscheinen. Sie bietet sich auch an, wenn sich die Bundesländer aufmachen, ihre regionale Verantwortung für den ambulanten und sektorunabhängigen Teil der Versorgung zu stärken. Die Länder können schwerlich bundesweite Richtlinien und Regelungen auf die Anwendung in ihrem Land hin moderieren und beaufsichtigen, wenn sie selbst solche Regelungen in nicht nachvollziehbarer Weise abwehren.

Damit verbunden sein sollte, dass sich die Länder konsequenterweise auf eine Rahmenplanung konzentrieren, die Richtlinien des G-BA auf das Land anwendet, während die konkrete $\mathrm{Zu}$ lassungs- und Vertragshoheit auf die Krankenkassen übergeht. Im Interesse der Patienten sind folgende Anforderungen zu erfüllen:

\section{Erreichbarkeit einer qualitativ de-} finierten Notfallversorgung: Hier bietet sich der Rückgriff auf die in den Rettungsdienstgesetzen der Länder festgelegten Hilfsfristen an. So wird im Hessischen Krankenhausrahmenplan die Notfallversorgung als enge Verzahnung zwischen Rettungsdienst und Krankenhaus beschrieben. Die Regelentfernung zwischen Notfallort und Krankenhaus sollte 15 bis $25 \mathrm{~km}$ $\operatorname{sein}^{26}$. Somit ergeben sich rechnerisch ca. $35 \mathrm{~km}$ als Entfernung zwischen Notfallkrankenhäusern. Die oben beschriebene enge Verzahnung ergibt sich schon aus der Tatsache, dass die Notärzte in der Regel von den Krankenhäusern gestellt werden.

2. Festlegung von Krankenhausstandorten für dringliche und Routinebehandlungen: Nach der Häufigkeit (ICD-10 Code) zählen dazu aus den Fachgebieten Gynäkologie/Geburtshilfe, Innere Medizin und Chirurgie die Beispiele Lebendgeburt, Herzinsuffizienz (Herzschwäche) und Cholelithiasis (Gallensteine). Die Diagnostik und Therapie dieser Zustände bzw. Erkrankungen erfordert eine personelle, räumliche und apparative Infrastruktur, die in der Regel im traditionellen mittelgroßen Kreis- krankenhaus vorhanden war und ist. Die Grenzen zur Routinebehandlung werden dann überschritten, wenn durch Komplikationen oder besondere Risiken entsprechend besondere Vorhaltungen notwendig werden. Das könnte bei den oben beispielhaft genannten Diagnosen die Reanimation von Frühgeburten, die Intensivbehandlung bei kardiogenem Schock bei Herzinsuffizienz oder eine Komplikation bei der Gallenblasenoperation sein.

\section{Freigabe von Krankenhäusern für} Selektivverträge unter Berücksichtigung der Qualität bei „planbaren Leistungen": Hier bietet sich ein Einstieg bei planbaren Eingriffen wie der Implantation von Hüft- und Kniegelenksendoprothesen an. Auf der Basis von Qualitätsbewertungsinstrumenten wie Daten über Infektionsraten oder Revisionseingriffe lassen sich P4P-Verträge schließen, die Wirtschaftlichkeitspotenziale unmittelbar mit hohen Qualitätsstandards verbinden $^{27}$. Bei der Erreichbarkeit der Krankenhäuser ist mit einer allgemein akzeptierten Fahrzeit von 1 Std. zu rechnen. Sie nimmt mit dem Verständnis von Qualitätsvorteilen für den Patienten sicherlich zu. Die Vergabe von Versorgungsaufträgen durch die Krankenkassen erfolgt auf der Grundlage von Ausschreibungen, wobei eine Mindestzahl von etwa drei zu berücksichtigenden Krankenhäusern erforderlich sein wird. ${ }^{28}$

4. Planung von Krankenhäusern der Zentral- und Maximalversorgung: Auch wenn Zentral- und Maximal-

24 Vgl. Michael Coenen, Justus Haucap und Annika Herr: Regionalität - wettbewerbliche Überlegungen zum Krankenhausmarkt. Krankenhausreport 2012 S. 152.

25 Für eine vergleichende Darstellung der Krankenhausplanungspraxis der Bundesländer vgl. Jürgen Malzahn, Christian Wehner und Claus Fahlenbrach: Krankenhausplanung in der Krise. Sicherstellung der Gesundheitsversorgung - Neue Konzepte für Stadt und Land, Berlin 2011, S. 44.

26 Hessischer Krankenhausplan 2009 S. 31

27 Jürgen Malzahn, Christian Günster und Claus Fahlenbrach: Pay-for-Performance - Einsparungen und Bonuszahlungen bei Hüft-EP-Implantationen. Krankenhausreport 2012 S. 255

28 Vgl. Thomas Göbel und Johannes Wolff: Direktverträge für stationäre Leistungen. Krankenhausreport 2012 S. 139 
versorger überwiegend Krankheitsbilder behandeln, die mit geringerem Ressourceneinsatz ebenso gut und wohnortnäher von Krankenhäusern der Grund- und Regelversorgung versorgt werden könnten, haben die Länder auf diese Krankenhäuser, wenn es um universitäre Medizin geht, ein besonderes Augenmerk. Das hängt auch mit Prestige und Ansehen zusammen, wenn es z. B. um Transplantationsmedizin oder Herzchirurgie geht.

Dennoch ist die Frage erlaubt, warum in Deutschland der weit überwiegende Teil von mehr als 30 Universitätskliniken Kinderherzchirurgie (teilweise mit erschreckend geringen Fallzahlen) betreibt. Die Schweden haben es geschafft, diese Chirurgie in einem Land mit großer Fläche in zwei Zentren zu konzentrieren, was zwar häufig lange Anfahrtswege, dafür aber aufgrund größerer Zahlen hinreichende Erfahrung bei der komplizierten Operation bedeutet. Auslöser für diese Konzentration war gerade auch der Elternwunsch herzkranker Kinder, ihr Kind gut versorgt haben zu wollen und dafür gern längere Anfahrtswege in Kauf zu nehmen.

Für den Bereich der Routinekrankheitsbilder und planbaren Eingriffe sollten sich die Länder auch in Deutschland auf eine qualifizierte Rahmenplanung und Aufsicht auf hohem Niveau konzentrieren. Diese Bereiche können dann schrittweise wettbewerblich kontrahiert werden. Für die universitäre und hoch spezialisierte Medizin verbliebe auch in dieser Perspektive eine abschließende Planungshoheit der Länder, die stärker als bisher Erkenntnisse der Versorgungsforschung einbeziehen und zur systematischen Innovationsbewertung beitragen sollte.

Und schließlich bleiben die wichtigen Fragen nach der Krankenhausinvestitionsfinanzierung bzw. der Einführung der Monistik und dem Verlauf der Grenze zwischen kollektivvertraglichen Regelungen und Selektivverträgen im Krankenhaus. Monistik würde den Wettbewerb sektorübergreifend beschleunigen und Anreize setzen, die auch den Selektivverträgen zugute kämen. Entscheidend dafür ist aber die Bereitschaft der Länder, im Verein mit Krankenkassen und innovativen Krankenhausträgern über die in Stein gemei- ßelten Rechte der Krankenhausplanung nachzudenken und bei der Investitionsfinanzierung neue Wege ins Auge zu fassen.

\section{Krankenhaus-Wettbewerb?}

Bei aller Zustimmung zu dem diagnosebasierten, leistungsorientierten Vergütungssystem im Krankenhaus (GDRG-System) bleiben für Krankenhausplanung und -Politik Fragen nach dem Versorgungsauftrag der Krankenhäuser und seinem ethischen Fundament. Die ökonomisch induzierte Expansion der Eingriffe wird inzwischen eingeräumt ${ }^{29}$ : Indikationsgrenzen werden gesenkt, Risiken für Patienten in Kauf genommen und eine aufmerksame, zuwartende Behandlung weicht dem möglichen Eingriff ${ }^{30}$. Es ist bemerkenswert, dass auch die Bundesärztekammer $^{31}$ inzwischen Zielvereinbarungsverträge mit leitenden Krankenhausärzten als unethisch kennzeichnet. Die BÄK wendet sich gegen Zielvereinbarungen der Häuser mit leitenden Ärzten, wie sie offenbar auch Transplantationsmediziner geleitet haben, die in Göttingen und Regensburg Befunde manipuliert hatten, um mehr Organe für ihre Patienten zu erlangen ${ }^{32}$. Generell werden immer mehr Organe an der Warteliste vorbei vergeben. Einen schleichenden Rückzug der Medizin auf ein „mehr Leistungen“ und das ökonomische Anreizsystem darf es nicht geben.

Eine Stimme lässt aufhorchen! Neben dem Sachverständigenrat, der der aktu-

29 Nach Angaben des StBA nahm die Zahl der Operationen allein von 2009 auf 2010 um 5,2 Prozent zu. Vgl. Pressemeldung Nr. 379 vom 12.10.11.

30 Aktuell wird etwa über die Häufigkeit von Prostata-Operationen in Deutschland diskutiert, vgl.: Ärzte operieren zu schnell bei Prostata-Krebs, in: Frankfurter Rundschau vom 24. Juli 2012.

31 Vgl. „Boni für Chefärzte - Bedenkliche Anreize“, in: Deutsches Ärzteblatt 2012; 109(7): A-298 / B-262 / C-258

32 Vgl. Berliner Zeitung vom 24. Juli 2012.

33 Vgl. Münch, Eugen: Industrialisierung der Medizin Netzwerkprozesse- ergänzende Bearbeitung: Dr. Boris Augurzky, RheinischWestfälisches Institut für Wirtschaftsforschung e.V. (RWI), in: Die Zukunft personenbezogener Dienstleistungen im Gesundheitswesen / IGES Institut GmbH, Vortrag am 18.4..2012 in Berlin.

34 Schönbach, K.H.: a.a.O., S. 17. ellen Politik immer eine Zeit voraus sein muss, gibt es ein Lebenszeichen vitalen Wettbewerbs aus der Privatwirtschaft. Die ins Stocken geratende Fusion von Rhön und Helios zum größten Krankenhauskonzern Deutschlands mit rund 80.000 Mitarbeitern hatte Eugen Münch ${ }^{33}$ als Spindoctor der Branche mit Überlegungen verbunden, eine $\mathrm{Ge}$ samtversorgung als Zusatzversicherung anzubieten: Wo „mehr Leistungen“ als Geschäftsmodell der PKV in Konkurs gehen, bietet qualitätsgesteuerte Medizin im Netzwerkverbund ein werthaltiges Leistungsversprechen. Eine Optimierung der Versorgung im Netzwerk 